\title{
Gestión de los expatriados. Elementos clave del proceso para las empresas en entornos internacionales
}

Management of Expats: Key Elements of the Process for Businesses in International Environments

Gestão dos expatriados. Elementos chave do processo para as empresas em entornos internacionais

\section{Yesenia Verónica Polanco Pantoja*}

Fecha de recibido: 18 de enero de 2017

Fecha de aprobado: 10 de julio de 2017

Doi: http://dx.doi.org/10.12804/revistas.urosario.edu.co/empresa/a.5327

Para citar: Polanco Pantoja, Y. V. (2018). Gestión de los expatriados. Elementos clave del proceso para las empresas en entornos internacionales. Universidad \& Empresa, 20(34), 103-126. Doi: http://dx. doi. org/10.12804/revistas.urosario.edu.co/empresa/a.5327

\section{RESUMEN}

Actualmente, la expatriación es una práctica común, llevada a cabo por las empresas que han logrado la internacionalización. En el presente artículo se realiza una revisión teórica sobre los elementos clave que deberían ser considerados por las empresas globales en cada una de las diferentes etapas de este proceso, con el fin de lograr que sea exitoso. En primer lugar, se expone la fase previa o arranque de la misión en el extranjero; después, se aborda

Doctora en Ciencias Empresariales por la Universidad Antonio de Nebrija, Madrid, España. Magíster en Ciencias de la Educación por la Universidad Nacional Autónoma de Nicaragua. Directora de la Facultad de Ciencias Administrativas de la Universidad Evangélica Nicaragüense (UENIC). Correo electrónico: cadministrativas@uenicmlk.edu.ni 
la fase de desarrollo y se concluye con la fase de repatriación o regreso del trabajador al país de origen. La revisión de la literatura realizada fue integrativa y sistemática. Para tal efecto, se analizaron y sistematizaron los más recientes estudios publicados por diferentes instituciones de investigación, en los que se consideraron múltiples empresas transnacionales en diferentes industrias.

Palabras clave: Expatriación, talento global, internacionalización, recursos humanos.

\section{ABSTRACT}

Expatriation is now a common practice for companies that want to achieve internationalization or have done so. In the present article, a theoretical review is made of the key elements that should be considered by global companies in each of the different stages of the expatriation process in order to successfully carry out expatriation. It begins with a previous phase or with the beginning of the international assignment. Next, the development phase is addressed, and the process concludes with the worker's repatriation or return to his or her country of origin. The review of literature is comprehensive and systematic. For this purpose, the most recent studies published by different research institutions were analyzed and systematized. These studies considered multiple transnational companies in different industries.

Palabras claves: Expatriation, global talent, internationalization, human resources.

\section{RESUMO}

A expatriação é na atualidade uma prática comum levada a cabo pelas empresas que desejam ou têm conseguido a internacionalização. No presente artigo realiza-se uma revisão teórica sobre os elementos chave que deveriam ser considerados pelas empresas globais em cada uma das diferentes etapas do processo de expatriação, com o fim de conseguir uma expatriação bem-sucedida. Inicia-se com a fase prévia ou de arranque da missão no estrangeiro. A continuação, aborda-se a fase de desenvolvimento e se conclui com a fase de repatriação ou regresso do trabalhador ao país de origem. A revisão da literatura realizada foi integrativa e sistémica. Para tanto, se analisaram e sistematizaram os mais recentes estudos publicados por diferentes instituições de pesquisa. Ditos estudos consideraram múltiplas empresas transnacionais em diferentes industrias.

Palavras-chave: Expatriação, talento global, internacionalização, recursos humanos. 


\section{INTRODUCCIÓN}

Como resultado de la globalización de la actividad económica, las empresas que procuran la internacionalización o la han logrado se enfrentan al reto de movilizar por un tiempo determinado a parte de su fuerza de trabajo cualificada para realizar tareas específicas en el extranjero. Como es de suponer, la expatriación es más usual en organizaciones de gran tamaño que en pequeñas y medianas empresas. El desplazamiento o movilidad internacional implica para la firma una serie de acciones críticas antes, durante y después de la expatriación y para el expatriado este traslado temporal a un país diferente al lugar de donde fue contratado trae una serie de afectaciones a su ámbito social y familiar. El desplazamiento significa un reto de grandes magnitudes sociales, culturales y económicas y es por ello que deberá afrontarse con una visión global.

Considerando los altos costos económicos que representa para la organización una misión internacional fracasada, este proceso requiere una gestión profesional que facilite el aprovechamiento de todas sus ventajas y permita alcanzar los objetivos. Los expatriados son recursos humanos clave en el desarrollo de la firma, por lo que su dirección debe llevarse a cabo de manera diferenciada en comparación con el resto de los miembros de la organización. La empresa debe tomar conciencia de que gestiona un recurso valioso que juega un papel estratégico fuera de las fronteras de la oficina matriz; debido a ello, la gestión del proceso de expatriación se convierte en un factor de vital importancia y de ésta dependerá en gran medida el éxito de la asignación internacional.

Las altas exigencias actuales para los Departamentos de Recursos Humanos -quienes deben dotar a las empresas multinacionales con el talento adecuado en el momento en que se necesita- convierten la gestión de expatriados en una función cada vez más crítica y difícil. A medida que las empresas tienen que trasladar empleados a través de las fronteras para promover el crecimiento global la movilización del talento, se han convertido en un factor clave para el éxito general de la organización.

El hecho de que de manera creciente las empresas recurren a la expatriación, ha convertido el tema en un tópico de gran interés y de obligatorio abordaje cuando se analiza la gestión del talento global.

Tomando en consideración los elementos antes planteados, el presente artículo tiene como propósito 
exponer los factores críticos a considerar durante cada una de las etapas del proceso de expatriación y a partir del análisis se plantean aspectos claves que deberían atender los Departamentos de Recursos Humanos y la empresa en su conjunto, para lograr la consecución exitosa de los objetivos en las misiones en el extranjero.

\section{REVISIÓN DE LITERATURA}

La crisis económica iniciada en 2008 ha provocado una mayor presión por reducir los costos de la movilización internacional; sin embargo, la expatriación continúa creciendo como vía para la ampliación de los negocios a nivel global y también porque se ha convertido para algunas empresas en una exigencia para asegurar la supervivencia.

De acuerdo con un estudio realizado por IESE-IRCO y ERES Relocation en el año $2009,{ }^{1}$ entre los años 2007 y 2008 , el $46 \%$ de las multinacionales españolas estudiadas habían aumentado el número de expatriados en un 33\%. Por otra parte, algo más de la mitad de las multinacionales extranjeras había aumentado el número de expatriados en España en un 37\%. Esta investigación muestra además que las perspectivas para el año 2009 apuntaban a un mantenimiento o crecimiento de dicha cifra.

El estudio Tendencias globales del capital humano $(2014)^{2}$ señala que las empresas que operan a nivel global, ofrecen a sus empleados de alto potencial asignaciones de desarrollo a nivel mundial como uno de sus viajes obligados. A su vez, estas firmas se enfrentan a la necesidad de mover especialistas a las misiones globales con rapidez.

Una investigación realizada en 2008 por el Instituto de Estudios Superiores de la Empresa IESE Business School y Ernst \& Young 3 confirma la tendencia de las empresas a promover la expatriación como práctica habitual. El 68,4\% de los expatriados consultados laboraban en organizaciones con más de 5.000 empleados. A éstos se les preguntó acerca del número de expatriados de su empresa. Los resultados se muestran en la figura 1 .

1 En el estudio participaron 30 empresas multinacionales. De éstas, 14 eran españolas y 16 extranjeras, de diferentes sectores.

2 El estudio fue realizado con un total de 2.532 empresas y líderes de recursos humanos en 94 países.

3 El estudio se realizó con una muestra de 510 expatriados de diferentes compañías. 
Figura 1. Número de expatriados de su empresa

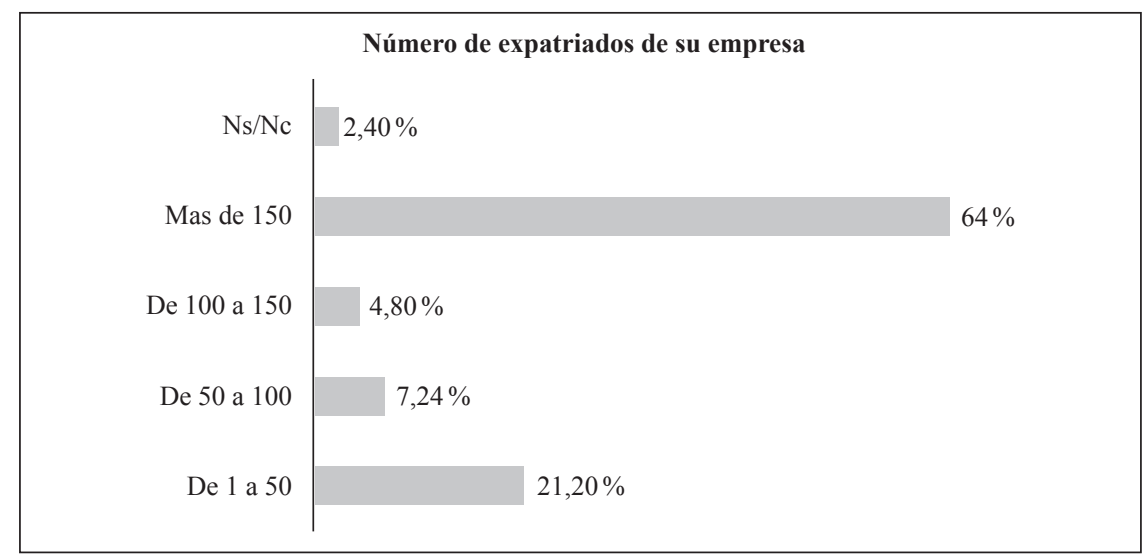

Fuente: IESE y Ernst \& Young (2008).

Como puede observarse, el $64 \%$ de los encuestados afirma que su empresa ha expatriado a más de 150 colegas.

Por su parte, BGRS, NFTC y CGHB $(2015)^{4}$ revelan una nueva tendencia y es que cada vez más expatriados realizan estadías de periodos más cortos. En 2013, el 18\% de los expatriados había estado en cinco o más países. En 2015, el porcentaje ascendía a $25 \%$.

De acuerdo con Wortmann (2014), en el marco de la globalización de los mercados y las empresas, la mo- vilización de personal hacia el extranjero -conocida como expatriación - ha cobrado alta relevancia. Considerando los datos aportados por las diferentes organizaciones mencionadas anteriormente, se puede afirmar que este proceso está en aumento.

\section{1. ¿Qué es un expatriado?}

De acuerdo con Porret (2014) expatriado es el empleado de una compañía que se desplaza, por un tiempo más o menos prolongado, de un país a otro bajo un programa específico (decisiones y acuerdos internos) y

$4 \quad$ El estudio fue realizado por la empresa Brookfield Global Relocation Service (BGRS, por sus siglas en inglés), y patrocinado por el Consejo Nacional de Comercio Exterior (NFTC, por sus siglas en inglés) de Estados Unidos y la compañía de seguros de salud Cigna Global Health Benefits (CGHB, por sus siglas en inglés). En el estudio participaron un total de 2.704 expatriados que se encontraban en asignaciones en 156 países alrededor del mundo. 
que está de acuerdo con la nueva situación en que se encontrará, teniendo en cuenta dónde estaba anteriormente y considerando una serie de circunstancias personales, profesionales, familiares, culturales, sanitarias, religiosas, económicas y de ocio.

Orsi, Bertoia y Barbosa-Lima (2015) plantean que la expatriación es un proceso caracterizado por la transferencia de ejecutivos de la empresa matriz a las subsidiarias o de una subsidiaria a otra. Por su parte, Mondy (2010) considera que un expatriado es un empleado que no es ciudadano del país en el cual se localizan las operaciones de una empresa, pero es ciudadano de la nación en la cual la organización tiene sus oficinas centrales. Así, un expatriado es un trabajador más de la empresa asignado a una misión especial en el extranjero por un tiempo limitado.

\section{ESTRATEGIA METODOLÓGICA}

La presente investigación es de tipo descriptivo, dado que se realizó una revisión exhaustiva de la literatura relativa a la temática, esto es así porque se buscaba evaluar y detallar el estado actual de las estrategias utilizadas por las transnacionales en cada una de las tres etapas del proceso de expatriación, con el fin de proponer elementos a tener en cuenta para llevar a cabo una gestión exitosa de los expatriados. Los estudios de tipo descriptivo "miden o evalúan diversos aspectos, dimensiones o componentes del fenómeno o fenómenos a investigar" (Hernández, Fernández \& Baptista, 2010, p. 61).

Así mismo, se utilizó el método cualitativo porque se procedió a analizar diferentes investigaciones realizadas en el campo de la expatriación, específicamente en las diferentes etapas del proceso de expatriación de trabajadores en empresas transnacionales, con el fin de comprender mejor las estrategias aplicadas por las firmas. De acuerdo con Báez y Pérez y Tudela (2009) "el objeto del método cualitativo es el conocimiento de la realidad" (p.15).

La revisión de la literatura realizada fue integrativa y sistemática; para tal efecto se analizaron y sistematizaron los más recientes estudios publicados por diferentes instituciones de investigación. Dichos estudios consideraron múltiples empresas transnacionales en diferentes industrias, que cuentan con expatriados en diferentes países y continentes. Lo anterior, contribuyó a aportar una visión global de la expatriación para proceder a seleccionar los elementos de interés específicos en la investigación. 


\section{RESULTADOS}

\subsection{Fases del proceso de expatriación}

De acuerdo con IESE y Ernst \& Young (2008), la expatriación consta de tres fases, La primera es la fase previa o inicio, en la que se consideran diversas políticas y prácticas como selección, comunicación, asesoramiento y retribución, conforman la propuesta de la empresa e inciden, así mismo, en la valoración y posterior decisión de aceptar o no la expatriación por parte de los candidatos.
Una vez que la propuesta es aceptada, en el país de acogida se da inicio a la fase de desarrollo, en la que se consideran aspectos relacionados con la adaptación, la consecución de expectativas personales, el desarrollo profesional y el reconocimiento de la empresa. Finalmente, se concluye el proceso con la fase de repatriación, que incluye la readaptación en el país de origen, beneficios posteriores y oportunidad de promoción dentro de la empresa, entre otros.

\section{Figura 2. Fases del proceso de expatriación}

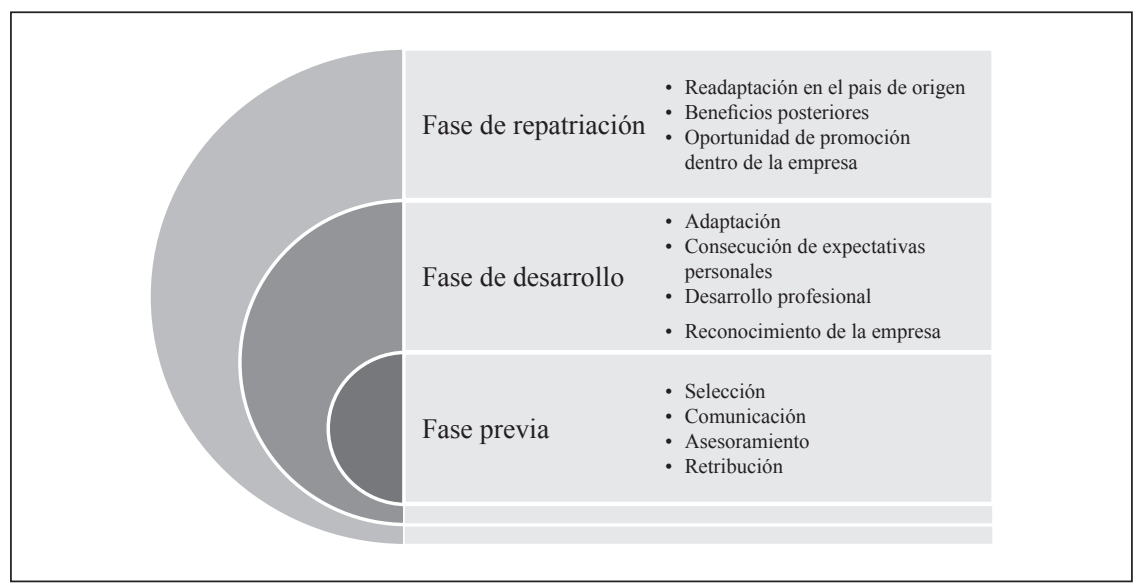

Fuente: elaboración propia, a partir de IESE y Ernst \& Young (2008). Según Brookfield Global Relocation Service (2016), ${ }^{5}$ entre los principales desafíos de gestión en las asignaciones internacionales se encuentran cuatro factores críticos y difíciles de gestionar: cumplimiento/gestión de riesgos, contenido de los costos, ajuste del expatriado/familia en el país de destino y administración total de la complejidad del programa de movilidad. Cada una de las tareas antes mencionadas tiene sus propias particularidades, lo que eleva el nivel de exigencia para el área de recursos humanos.

5 El estudio se conoce como Informe de Movilidad Internacional y fue realizado entre 163 empresas en 72 países y corresponde a los resultados de la edición número 21 de la encuesta anual de movilidad Global Trends. 


\subsubsection{Fase previa o inicio}

\subsubsection{Elementos clave en la} selección del candidato idóneo para una asignación internacional

Esta fase empieza con la selección del personal adecuado para llevar a cabo la misión. Normalmente se espera que la empresa haya identificado con anterioridad a los posibles candidatos por su capacidad y potencial de desarrollo. Sin embargo, algunos estudios muestran que el Departamento de Recursos Humanos debería esforzarse más en esta fase. De acuerdo con el Informe de Movilidad Internacional (2016), el 73\% de las 163 empresas que formó parte del estudio no mantenían una lista de candidatos para futuras misiones internacionales y el $33 \%$ no contaba con un proceso para que los empleados se designasen a sí mismos como dispuestos a aceptar una asignación internacional. Lo anterior debería constituir una voz de alerta, puesto que éste componente del proceso es de vital importancia, ya que de no seleccionarse a la persona idónea para esta tarea, el resto del proceso no será llevado a buen término.

Al momento de la selección deben tomarse en cuenta no solamente los requerimientos y necesidades del cargo, sino otros elementos que podrían incidir en el éxito de los expatriados en el país de destino. Según el Informe de Movilidad Internacional (2016), el 78\% de las empresas estudiadas no evaluaba formalmente la capacidad de adaptación de los candidatos y solamente el 29\% utilizaba algún tipo de herramienta de evaluación. Con estos hallazgos se puede afirmar que en la actualidad las empresas afrontan una serie de retos en diferentes frentes, lo que abre el espacio para nuevas áreas de mejora, a fin de llevar a cabo una gestión de la expatriación de forma exitosa. Dados los conocimientos aportados por los estudios realizados hasta la fecha, se puede ampliar el campo de conocimiento con miras a afrontar mejor estas cuestiones en futuras asignaciones internacionales. Considerando lo anterior, cabe preguntarse, ¿qué es lo que debe tener en cuenta la empresa para seleccionar al personal idóneo para las asignaciones internacionales?

En cuanto a la selección, los criterios a utilizar deben contemplar "tres aspectos fundamentales: cualificación profesional o técnica, habilidades directivas y liderazgo, y sensibilidad hacia la cultura del país de destino" (Rodríguez Candela, Fernández \& Romero, 2013, p. 386). Otros autores como Brayer Hess y Linderman (2007) apuntan que las competencias clave con las que debe 
contar un futuro expatriado, son las siguientes:

- Habilidad para tolerar la frustración, la ambigüedad y los errores.

- Apertura mental y tolerancia a las diferencias.

- Flexibilidad y adaptabilidad.

- Curiosidad y disfrute por nuevas experiencias.

- Buenas habilidades de comunicación y observación.

- Autosuficiencia y un fuerte sentido de sí mismo.

Como se puede observar, el tipo de habilidades requeridas van más allá del dominio de los aspectos meramente técnicos, debido a que "deben tenerse en cuenta los factores económicos, legales, políticos, migratorios, laborales, sicológicos, familiares y socioculturales que configuran las distintas realidades de las personas implicadas, y de los lugares de origen y destino" (Gadow, 2010, p. 285).

El estudio de Ernst \& Young (2008), concluye que la mayoría de los expatriados considera que las empresas durante su proceso de selección tomaron en cuenta:

- Conocimientos técnicos y experiencia.

- Consideración de la carrera profesional.

- Capacidad de adaptación cultural.
- Capacidad de liderazgo.

- Habilidades negociadoras.

Una vez hecha la valoración pormenorizada de estos elementos debería elegirse al candidato mejor calificado y al que presente la mayor disposición para asumir su labor en este importante proceso.

El candidato a la expatriación podría plantearse una serie de interrogantes, puesto que normalmente se generan muchas inquietudes frente a un posible cambio de destino, un nuevo empleo y todo lo que esto implica. Por ejemplo, el trabajador podría preguntarse: ¿estoy dispuesto a irme a trabajar al extranjero?, ¿qué tan positiva sería esta experiencia para mi desarrollo profesional?, ¿podré adaptarme al país de acogida?, ¿mi familia podrá lidiar con el nuevo entorno?, ¿se me da bien el manejo del idioma extranjero?

Si al finalizar este balance personal se concluye con la mayor cantidad de respuestas positivas, es probable que el empleado acepte la asignación internacional.

\subsubsection{Retribución y apoyos para el expatriado}

Al ofrecer la oportunidad de la movilidad internacional, la empresa deberá proponer una "maleta para 
la expatriación", que contenga todas las ayudas/promociones para llevar a cabo la misión en el extranjero.

Es importante destacar que este paquete debe ser interesante y ofrecer el atractivo suficiente para conquistar a los empleados calificados. En este sentido, la tendencia, de acuerdo con el Informe Movilidad Internacional (2016), es que las empresas cada vez más ${ }^{6}$ realizan esfuerzos para reducir los costos de movilidad en respuesta a las condiciones económicas actuales; esto también es señalado por IESE y Ernst \& Young (2008) cuando afirman que las políticas otrora generosas, se están restringiendo.

Sin embargo, las firmas deben contribuir a la movilidad del empleado con una política de remuneración $\mathrm{y}$ beneficios que sean uniformes para todos. "La compensación y los beneficios de los directivos expatriados deben tener un fin fundamental: apoyar los intereses estratégicos de la empresa, alineándolos con los propios intereses de los empleados" (Jiménez, 2013, p. 67). Por un lado, se debe asegurar la efi- ciencia desde el punto de vista de los costos para la compañía, pero también la compensación habrá de ser razonable y en concordancia con los niveles del país de destino. Así mismo, es necesario ser competitivo en comparación con las prácticas de otras multinacionales.

Un elemento esencial en este punto lo constituye la comunicación. Es imprescindible que la organización le dé al expatriado una explicación clara y detallada de los beneficios y ayudas que recibirá antes de iniciar la misión; de esta forma, se evitarán malentendidos posteriores que podrían generar ruido en la relación, un posible desencanto $\mathrm{y}$, en última instancia, un aborto de la misión.

También es común que muchas empresas ofrezcan asesoramiento previo a la mudanza definitiva, pero lo ideal sería que estos apoyos se brindaran en dos momentos: ex ante expatriación y ex post expatriación. De acuerdo con López (2014), las ayudas pueden ser de diversa naturaleza y se deben efectuar en cada etapa del proceso (tabla 1).

6 El 69\% de las empresas participantes en el estudio afirma realizar esfuerzos para reducir los costos de la movilidad de sus empleados. 
Tabla 1. Ayudas que podrían otorgar las empresas a los expatriados durante cada una de las fases del proceso

\begin{tabular}{|l|l|l|}
\hline \multicolumn{1}{|c|}{ Ayudas previas } & \multicolumn{1}{|c|}{ Ayudas en destino } & \multicolumn{1}{c|}{ Ayudas de repatriación } \\
\hline Comunicación & Adaptación & Regreso \\
\hline Formación & $\begin{array}{l}\text { Asesoramiento jurídico-laboral, } \\
\text { seguridad social y fiscal }\end{array}$ & Ampliación del plazo \\
\cline { 1 - 2 } $\begin{array}{l}\text { Información previa del } \\
\text { país destino }\end{array}$ & $\begin{array}{l}\text { Adaptación personal, familiar y } \\
\text { profesional }\end{array}$ & Cambio de empresa de origen \\
\hline $\begin{array}{l}\text { Información sobre } \\
\text { estilo de vida }\end{array}$ & & \\
\hline $\begin{array}{l}\text { Información sobre el } \\
\text { puesto de trabajo }\end{array}$ & $\begin{array}{l}\text { Si contará con } \\
\text { asesoramiento: fiscal, } \\
\text { laboral, etc. }\end{array}$ & \\
\hline $\begin{array}{l}\text { Si contará con ayudas } \\
\text { al cónyuge }\end{array}$ & & \\
\hline
\end{tabular}

Fuente: López (2014).

Desde la óptica de los expatriados, y de acuerdo con el estudio de IESE y Ernst \& Young (2008), los factores que reciben una mejor valoración son las ayudas en el tiempo de planificación de la expatriación, en orden de importancia: el número de viajes previos necesarios al país de destino, información, trabajo del cónyuge o la adaptación familiar.

Respecto a las ayudas en destino, debe prestársele especial atención a los impuestos. IESE y Ernst \& Young (2011) señalan que la mayor parte de los encuestados $(97,6 \%)$ ha contado con asesoramiento fiscal especializado facilitado por la empresa. Por otra parte, algunas compañías cuentan con una "política de ecualización fiscal que consiste en deducir del salario los impuestos hipotéticos que un profesional destinado en el exterior tendría que pagar en su país de origen. A cambio, la empresa se hace cargo de todos los impuestos que le corresponde pagar en el país de destino" (IESE y Ernst \& Young, 2008, p. 14).

Dado que la mayoría de las firmas multinacionales no cuentan con un departamento especializado en expatriación (IESE-IRCO \& ERES Relocation, 2009), se puede recurrir a empresas especializadas que poseen este tipo de know-how y ofrecen asesorías en tópicos relativos a la gestión de expatriados. Algunos servicios en los que las empresas podrían requerir asistencia externa son asesoramiento en el diseño y 
desarrollo de políticas de protección fiscal del expatriado; diseño y desarrollo de políticas retributivas, cálculos impositivos y estimaciones fiscales, con el fin de determinar los costos impositivos generados por la expatriación; salario neto del expatriado, asesoramiento integral en el traslado y gestión del desplazamiento y coaching, entre otros.

De la buena preparación y comunicación por parte del Departamento de Recursos Humanos en cada uno de estos aspectos dependerá, en gran medida, la confianza y tranquilidad del trabajador para asumir la decisión de desplazarse.

\subsubsection{Fase de desarrollo}

Esta etapa da inicio en el momento en que el expatriado y su familia se han trasladado al país de destino; allí es donde comienza su integración en el entorno laboral y social, y por ende, es crucial que la empresa se esfuerce por hacer sentir respaldado al empleado. En esta etapa del proceso es fundamental el contacto y la comunicación con la organización, por eso se requiere especial esmero por parte de la firma para mantener y fortalecer el vínculo, y a la vez ser proactiva para favorecer al máximo la integración. El departamento de Recursos Humanos debe ser consciente de que el expatriado supone "entre 1,5 y 4 veces el gasto que representa un empleado local" (Espinosa, 2009, p. 46). La expatriación es una inversión que no se debe arriesgar y por lo tanto se debe prestar la debida atención a todos los detalles.

\section{1.2.1. Retos que genera la expatriación}

a. Retos en el plano familiar

Los posibles retos para el expatriado pueden generarse por diferentes frentes. De acuerdo con Rodríguez Candela, Fernández y Romero (2013) pueden ocurrir problemas de índole personal relacionados con el cónyuge o los hijos.

Por eso, durante la fase de desarrollo tienen gran influencia dos elementos fundamentales: por un lado, el cónyuge, y por otro, el ciclo de vida familiar en el que se encuentra el expatriado en el momento del desplazamiento. Según Storti (2007) varios estudios han encontrado que la razón más común por la que los expatriados no cumplen con eficacia una misión en el extranjero se debe a la incapacidad de su pareja para adaptarse al nuevo entorno. Las organizaciones no pueden olvidar que esta juega un rol esencial para el éxito de la expatriación, pues podría ser la fuerza para que avance el proceso 
o podría lograr que la marcha se paralice. "Por regla general, la mujer está más dispuesta a seguir a su cónyuge en un proceso de expatriación que al contrario" (IESE-IRCO \& ERES Relocation, 2009, p. 8).

Así mismo, el hecho de que los hijos sean pequeños y la pareja se dedique exclusivamente al hogar tiene efectos diferentes, tanto en la vida familiar como en la adaptación al nuevo entorno, en comparación con casos en los que los hijos son adolescentes y han construido fuertes lazos de amistad con vecinos, compañeros de clase y otros miembros de la comunidad. En este caso, podrían llegar a sentirse desarraigados en un momento muy complejo de su vida.

También pueden surgir muchas dificultades cuando el cónyuge es profesional y tiene que renunciar a su empleo para embarcarse en esta aventura. En esta situación, la pareja puede tener una serie de expectativas profesionales que podrían verse, de momento, truncadas por el cambio de residencia. El estudio llevado a cabo por IESE y Ernst \& Young (2011) señala que el 51,2\% de los cónyuges de los expatriados tiene una carrera universitaria, por lo que están calificadas para desenvolverse en el mercado laboral. Otro estudio realizado en el año 2008 por IESE y Ernst \& Young sostiene que las principales dificultades que en la actualidad se pueden apreciar en el ámbito de la expatriación son las relativas a las carreras duales: cada vez más, ambos cónyuges tienen expectativas de desarrollo y promoción profesional, y es muy complejo gestionarlas a la vez.

Generalmente, el expatriado tiene un horario laboral, supongamos de 8 a.m. a 5 p.m., en el que debe enfocarse y hacer frente a las nuevas expectativas laborales que se tienen de él. Por esta razón, la pareja es la que pasa más tiempo en contacto con la gente y la cultura del país, es decir, es la que vive más de cerca la expatriación; de no prestársele la atención adecuada, se podría llegar a desatender a esa persona, lo que acarreraría consecuencias como provocar que se sienta sola, con mucho tiempo a su disposición y sin mayores contactos sociales; esto último podría afectar a los miembros de la pareja cuando alguno de los dos no domina el idioma del país. Lo anterior podría dar a lugar a sendas presiones que, conjugadas con un alto nivel de estrés, desembocarían en una expatriación problemática, en una renuncia temprana a la expatriación o, en última instancia, a poner fin a la relación; según Fernández y Junquera (2014), el número de divorcios entre los expatriados es alto; por su parte, el Informe 
de Movilidad Global (2016) señala que los problemas relacionados con la familia son la causa principal de fracaso de las asignaciones internacionales.

Por esta razón, las empresas deberían afrontar mejor las cuestiones relativas al bienestar del cónyuge del expatriado, pues esta es una de las áreas de mejora que más atención debe tener durante el proceso de expatriación de personal.

b. Retos en el plano social y cultural

A menudo los expatriados y sus familias sufren por el choque cultural, producto de la confrontación que se da entre su cultura y la del país de destino. La interacción puede crear ambigüedad, problemas de comunicación y necesidad de aprender a desenvolverse en esa nueva cultura. "Es una experiencia totalmente normal cuando uno empieza a vivir en un país extranjero. La comida, el clima, las normas de conducta, todo es diferente" (Marinelli \& Fajardo, 2015, p. 461).

Los expatriados se desenvuelven ahora en un nuevo contexto intercultural, en el que interactuan con personas y organizaciones culturalmente distintas, por lo que podrían llegar a experimentar sentimientos de inquietud, irritabilidad e incompetencia (Hart, 2012), pues un nuevo tipo de entorno y situaciones inesperadas generan estrés. Cagigas y Arribas (2012) sostienen que durante su periodo de adaptación al nuevo país, cultura, usos y costumbres, todos los expatriados y sus familiares estarán expuestos a padecer diversos síntomas físicos -dolores de cabeza, alteraciones del ritmo cardíaco, aumento de la tensión muscular, cansancio, molestias digestivas- $\mathrm{y}$ de tipo síquico -tristeza, disminución del autocontrol emocional, excitabilidad e impaciencia-. Otros autores plantean que el choque cultural provocará en los expatriados "síntomas de inestabilidad emocional, impaciencia, pérdida de apetito, insomnio y leves alteraciones sicosomáticas" (Comelles, Allué, Bernal, Fernández-Rufete, \& Mascarella, 2009. p. 76). Esta sintomatología se presentará con mayor frecuencia cuanto mayor sea el contraste entre el país de origen y el país de destino.

De acuerdo con Espinosa (2009), normalmente un expatriado tiene que comunicarse, negociar, etc., con personas que tienen costumbres e idiosincrasia diferentes de la suya, $\mathrm{y}$ debe hacer frente a diversas situaciones de índole comercial y social. En estas circunstancias puede sentirse inseguro y desubicado, porque, al no conocer ampliamente las 
costumbres del país al que ha llegado, puede actuar o expresarse de una forma que podría ser considerada incorrecta o descortés para el interlocutor.

Según el Informe de Movilidad Internacional (2016), los países que mayor reto representan para los expatriados y sus familias son China, Brasil, India, Rusia y Arabia Saudita. Los expatriados señalan desafíos relativos a la calidad de vida, la contaminación del aire, la calidad de los alimentos, el clima, el acceso a la asistencia sanitaria, los costos de adaptación al entorno, preocupaciones por la seguridad, el lenguaje, el acceso a las escuelas internacionales de idiomas y barreras/diferencias culturales, entre otros.

Es aún más difícil cuando por cuestiones culturales la expatriación puede llegar a ser interpretada como una especie de exilio. Maison (2013) señala que la expatriación representa un desafío extra para las culturas latinas, tan enraizadas a sus costumbres y a la familia como núcleo de socialización.

También es necesario tener en cuenta que pueden darse problemas de adaptación al nuevo lugar de trabajo, a los colegas, al jefe, a diferentes estilos de trabajo, nuevos horarios, etc. Un temor que se cierne normalmente sobre los expatriados es lo relativo a no poder alcanzar los objetivos del proyecto en el tiempo planeado o a no alcanzarlos definitivamente; el sólo hecho de pensar en la posibilidad del fracaso profesional y personal genera estrés.

Afortunadamente, es posible superar estos desafíos. De acuerdo con Chow de la Peña y Molina Valdez (citados por Espinosa, 2009), el expatriado atraviesa una serie de etapas hasta que consigue una estabilidad. Al comienzo, todo el cambio le parece interesante y se siente atraído por el nuevo entorno; pasados dos o tres meses, la persona empieza a percibir el choque cultural y poco a poco comienza a sentirse como un extraño en ese ambiente; después de un tiempo deja de comparar su situación presente con la del pasado y comienza a conseguir la estabilidad necesaria para sentirse cómodo en el entorno en el que vive; en ese momento emprende cosas nuevas y es capaz de encontrar nuevos modelos de referencia. 
Figura 3. Transición del reajuste intercultural según $\mathbf{A l i}^{7}$

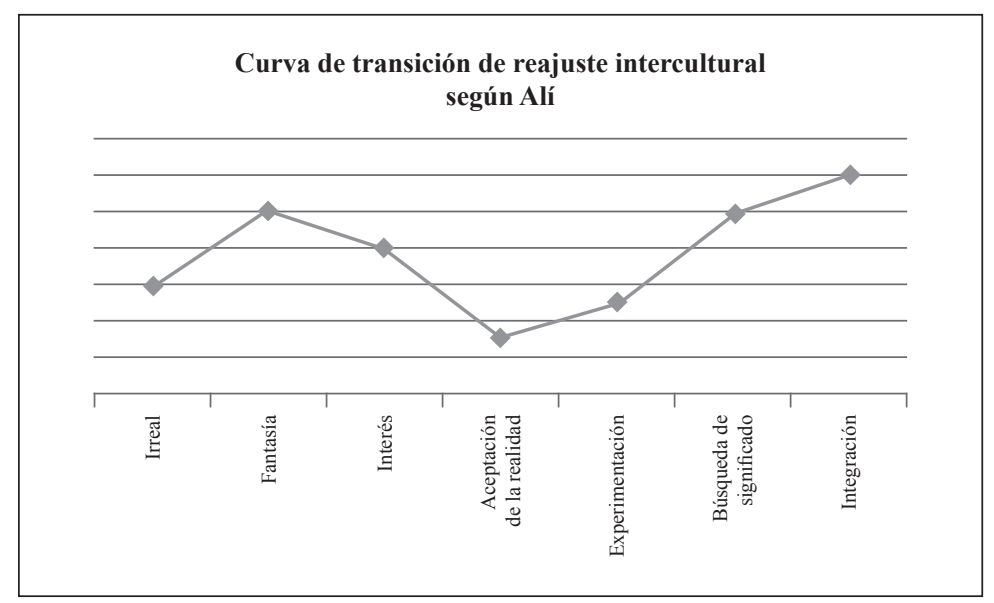

Fuente: Chow de la Peña y Molina Valdez (2004).

Considerando todos los retos que el expatriado y su familia deben enfrentar, es importante que la empresa ofrezca el apoyo necesario para hacer esta etapa lo menos traumática posible. La formación de lazos sociales debe ser un área crítica sobre la que el Departamento de Recursos Humanos debe prestar especial interés, puesto que éstos pueden sustituir en algunos casos los lazos familiares que los trabajadores dejaron atrás. El establecer vínculos con personas del país de acogida puede disminuir el estrés que sufren los expatriados y a reducir el periodo de adaptación.

\subsubsection{Programas de apoyo en multinacionales para facilitar el proceso de adaptación}

Algunas empresas ofrecen programas para facilitar el conocimiento de la cultura del país de acogida y el idioma. Autores como Baldwin, Bommer y Rubin (2015) señalan que Pepsi Co. cuenta con dos programas muy populares de entrenamiento y con beneficios para los familiares de los expatriados; por

7 Como lo muestra la figura 3, en la primera fase el expatriado tiene la sensación de que la reubicación es un sueño. En la fase siguiente, se produce un sentimiento de emoción debido al nuevo entorno. Seguidamente, profundiza la exploración en el desarrollo y la realización de los hechos. A continuación, "deja ir" las aptitudes del pasado confortable, es decir, supera la percepción de que es un extraño. Luego, comienza a tratar de hacer cosas diferentes. Después, entiende las razones del éxito o del fracaso, y crea nuevos modelos y teorías. Finalmente, aceptade un nuevo entorno. 
ejemplo, ofrecen entrenamiento en idiomas para mejorar la integración de los profesionales en el nuevo ambiente. La empresa también facilita soporte y ayuda financiera para procurar nuevas escuelas para los niños. Los cónyuges de los expatriados que no trabajan reciben ayuda para encontrar actividades no remuneradas (trabajo voluntario o estudios). En el caso específico de los expatriados de Pepsi en Ciudad de México, los trabajadores pueden participar en clases de zumba en las instalaciones de la empresa, por medio del programa de ejercicios físicos que utiliza danza latina; en Dubai, los funcionarios internacionales participan en olimpiadas de fútbol corporativas, y en China, en torneos de ping pong que los ayudan a relacionarse socialmente con los funcionarios locales.

Kimberly Clark, tiene 42.000 empleados en 56 países y además cuenta con programas de expatriación y asignaciones temporales de corta duración (por 3 a 6 meses) para la ejecución de proyectos en los diferentes países donde opera. Inicialmente, ofrecen apoyo logístico y operativo, pero luego las ayudas se expanden hacia un apoyo emocional para el expatriado y su familia.

Otro ejemplo de estos programas de apoyo planteado es el de PwC, la consultora con presencia en 157 países y 208,000 colaboradores (Baldwin, Bommer, \& Rubin, 2015). La firma cuenta con un programa de movilidad global que va desde los tres meses hasta los tres años, y ofrece apoyo a través de clases de idioma y otros datos básicos del lugar de destino. Como puede observarse, cada firma cuenta con programas específicos de apoyo a los expatriados, con miras a facilitar la adaptación en el país y la cultura de destino.

Programas como los mencionados anteriormente podrían aminorar las dificultades propias de esta fase; una vez cumplidas las tareas que dieron origen a la asignación internacional, el expatriado está listo para la última del proceso.

\subsubsection{Fase de repatriación}

Generalmente esta fase implica el retorno del expatriado a su país de origen una vez ha concluido la misión internacional, aunque también puede ocurrir que se dé antes del tiempo previsto, debido a los problemas mencionados anteriormente; en ese caso, el expatriado o la empresa deciden abortar la misión sin haber cumplido con los objetivos preestablecidos.

Esta fase tampoco es fácil de gestionar por parte del Departamento de 
Recursos Humanos, el expatriado y su familia porque, aunque marca el fin de la misión también puede presentar inconvenientes que dificultarían el cierre del proceso.

\subsubsection{Principales problemas}

De acuerdo con Storti (2003), la mayoría de los expatriados encuentran el ajuste de vuelta a casa, comúnmente conocido como choque cultural inverso, más difícil que el ajuste en el país de destino. "Quizás lo que más extrañan los retornados de estar en el extranjero es el sentido de la aventura y la emoción, la estimulación de estar rodeado de todo lo que es nuevo y diferente" (Storti, 2003, p. 78). Además, la vuelta a casa implica una serie de retos, que serán más manejables en la medida en que la empresa esté preparada con suficiente antelación para aplicar las políticas respectivas, y el expatriado conozca y haya aceptado los procedimientos administrativos sucesivos a su regreso. Algunas de las dificultades que se presentan en esta fase del proceso de expatriación obedecen a que al expatriado:

- La empresa no suele proveerle el estatus profesional que disfrutaba antes y comúnmente vuelve al puesto que desempeñaba previamente a su partida.

- No disfruta de las condiciones económicas que tenía en el país de destino: en su remuneración deja de percibir los complementos y las dietas.

- Las funciones en su rol laboral parecen ahora monótonas y menos interesantes.

Lo anterior se debe a que en la mayoría de los casos, la empresa no cuenta con políticas claras relativas a esta etapa. Según el Informe de movilidad internacional (2016), el $82 \%$ de las empresas consultadas no cuenta con una estrategia formal de repatriación vinculada a la gestión de carreras/planificación y la retención. Por su parte, IESEIRCO y ERES Relocation (2009) señalan que el $65 \%$ de las empresas estudiadas no cuenta con una política explicita de repatriación.

En este sentido, Storti (2003) afirma que los repatriados señalan la repatriación como la zona de mayor insatisfacción respecto a políticas de la organización. Entonces, ¿qué se demanda del Departamento de Recursos Humanos en esta fase?

Según el Informe de movilidad internacional (2016), las tres razones más comunes por las que los expatriados deciden abandonar la empresa después de la repatriación son, según su importancia: 1) que su nuevo rol en la organización no cumple con sus expectativas, 2) que 
considera que tiene mayor valor en el mercado de trabajo y 3) que no hay oportunidades dentro de la compañía para aplicar lo aprendido.

En el escenario actual, donde las habilidades críticas escasean, las empresas deben hacer un esfuerzo de más por desarrollar, retener y comprometer el capital humano que es cada vez más global. Los resultados de la encuesta de escasez de talento, realizada por ManpowerGroup en 2015 , revelan que el $38 \%$ de los más de 41.700 empleadores encuestados, provenientes de 42 países, están experimentando dificultad para cubrir puestos de trabajo, debido a la falta de talento disponible.

Con el fin de retener al expatriado como recurso clave y a la vez evitar vacios en la política de expatriación, es recomendable trabajar en los siguientes frentes:

- Mantener el apoyo al expatriado y a su familia. Como ya se ha enfatizado anteriormente, es necesario ayudar al trabajador y a su núcleo familiar para que tengan expectativas realistas antes de su regreso a casa, con el fin de que la transición sea menos traumá- tica. En la actualidad, la mitad de las empresas ${ }^{8}$ llevan a cabo la primera discusión con respecto a la planificación de la repatriación menos de seis meses antes de que ocurra.

- Elaboración de políticas claras que establezcan las medidas a tomar una vez que se haya producido la repatriación. De acuerdo con Arribas y Cagigas (2012), se requieren políticas concisas de repatriación, como un proceso ordenado en el tiempo que se anticipa a la fecha de regreso y un proceso transparente en cuanto a todas las opciones de colocación disponibles en cada momento. Se deben aclarar cuestiones relativas a ¿qué tratamiento profesional recibirá el empleado?, ¿se le ascenderá a su regreso?, ¿se le trasladará a un puesto distinto al que ocupaba antes de la asignación internacional?, ¿hallará cabida en la empresa?, ¿se le despedirá o el mismo renunciará?

- El conocimiento previo de la política de repatriación por parte del expatriado. Esto le permitirá al empleado que regresa despejar interrogantes sobre su futuro, propiciará un ambiente de mayor confianza y contribuirá a disminuir

8 De acuerdo con el Informe de movilidad internacional el 50\% aborda el tema de la repatriación menos de seis meses antes de que se produzca. El 28\% lo hace con más de seis meses de antelación, el $8 \%$ antes de la asignación inicie y el restante 14 no discute sobre este tema. 
la presión e incertidumbre que puedan sentir él y su familia.

El Informe de movilidad internacional (2016) señala que el $44 \%$ de las empresas encuestadas afirmó no contar con un programa o iniciativa para incrementar la retención de los expatriados. Este vacío conllevaría al desaprovechamiento de los expatriados como recurso valioso con talento global, que las empresas podrían estar dejando al margen sin detenerse a reflexionar sobre el costo que tuvo su experiencia internacional y sobre los beneficios que podrían representar para el futuro crecimiento y expansión de la organización en el mediano y largo plazo.

\section{1.3.2. Posibles áreas de mejora durante la fase repatriación}

A partir de lo señalado anteriormente, la empresa que ha utilizado la expatriación como práctica continua debe estar consciente de que ha ganado experiencia y posee personal con talento global, lo que le permite tener una ventaja competitiva frente a otras que no lo han hecho. Sus empleados son valiosos porque tienen la capacidad para desarrollar y ges- tionar proyectos y actividades estratégicas en contextos más amplios, y con su experiencia pueden enriquecer a sus equipos y a la firma en su conjunto. Quizá la compañía podría pensar en nombrarlo como mentor para los próximos expatriados.

Dessler (2004) provee algunas sugerencias a los Departamentos de Recursos Humanos para ayudar a reducir los problemas que afectan al expatriado a su regreso, por ejemplo: establecer contratos de expatriación, mantenerlo conectado con los negocios de la empresa matriz, ofrecer apoyo económico para conservar la casa del expatriado y ofrecer programas de re-inducción para él y su familia. Todas estas acciones contribuirían a promover el deseo de participación en misiones de expatriación para otros empleados que todavía no han sido considerados.

Por su parte, Arribas y Cagigas (2012) proponen que: la alta dirección debe participar en la formulación de la política de expatriación, sintiéndose responsable de su gestión.

ManpowerGroup (2014) ${ }^{9}$ en su informe "Gestión del talento" afirma que existe un consenso creciente

9 En el estudio participaron 2.200 líderes empresariales y profesionales de recursos humanos provenientes de 13 países y 24 sectores industriales. 
de que tener el talento adecuado en el momento preciso es responsabilidad de todos en la alta dirección.

- Las asignaciones internacionales deben ser una condición sine qua non para ser promovido a puestos directivos (en dependencia de la situación actual de la empresa).

- Los expatriados deben incluirse en los planes de sucesión de la empresa.

- Es necesario proveer formación al repatriado para facilitar su reinserción.

Por su parte, Hodgetts y Luthans (2003) señalan que para una repatriación exitosa es fundamental:

- Reconocer la repatriación como parte del proceso.

- Recibir de una buena forma al empleado y a su familia.

- Brindar la debida asistencia al repatriado para que tenga una buena integración familiar.

- Ayudar al cónyuge y a los hijos a su integración, por ejemplo, mediante la búsqueda de empleo o escuelas.

- Proporcionar un mentor al empleado para conocer de nuevo el mercado y la competencia.

- Revisar con el empleado y su cónyuge la expatriación que han tenido.

\section{CONCLUSIONES}

En la actualidad es frecuente que las multinacionales envíen a sus empleados clave alrededor del mundo para atender la creciente demanda de capital humano altamente calificado, requerido en el marco de la globalización. Esta ha impulsado a las empresas a extender el negocio a nuevos mercados en el extranjero o, dicho de otra manera, a internacionalizarse.

En este contexto, el proceso de la expatriación se ha convertido en una tarea crítica para el Departamento de Recursos Humanos y para la empresa en su conjunto. Los costos que implica para la firma una misión internacional fallida son altos, por lo que se requiere una gestión profesional de recursos humanos, que apunte al aprovechamiento de las ventajas de la expatriación y que conlleve al alcance de los objetivos. Sin embargo, la mayoría de las multinacionales no cuentan con el know how que se requiere en cada una de las fases de este proceso.

La primera fase del proceso demanda la atención de aspectos relativos a la selección del personal que va a participar en la expatriación, para la cual no se deben tener en cuenta solamente aspectos técnicos, sino habilidades directivas y de liderazgo, 
así como sensibilidad hacia la cultura del país de destino. Muchas empresas ofrecen diferentes tipos de ayudas al candidato seleccionado y a su familia antes del viaje, como, por ejemplo, visitas para conocer el país y cursos de idioma, entre otros.

La siguiente etapa o fase de desarrollo es crítica tanto para la empresa como para el expatriado. En esta el empleado entra en contacto con el nuevo entorno laboral y social, y es allí donde comúnmente se produce un choque cultural, que podría implicar para el expatriado y su familia una carga extra de estrés; normalmente se espera que al cabo de un tiempo ocurra una asimilación e integración al entorno. En esta etapa del proceso, la labor del Departamento de Recursos Humanos será dar un acompañamiento cercano al expatriado y a su familia para facilitar el logro de los objetivos de la misión.

Una vez cumplidas las tareas de la asignación, el expatriado regresará a su país; esto puede ser una experiencia estresante para él, por lo que la empresa debe contar con políticas claras al respecto, que faciliten la colocación del empleado en un área donde pueda hacer uso de su experiencia para el beneficio de la organización. Con esto se lograría, además, promover la retención de talento que constituya una ventaja competitiva para la organización.

Finalmente, debe enfatizarse que para lograr una exitosa gestión de la expatriación es de vital importancia la existencia de una política clara y transparente de expatriación que considere los factores clave en cada una de sus etapas y que otorgue al plano familiar alta prioridad.

\section{REFERENCIAS}

Baldwin, T., Bommer, W., \& Rubin, R. (2015). Gerenciandoo comportamento organizacional. O que os gestores eficazes sabem e fazem. Brasil: El Savier Editora Ltda.

Báez, J., \& Pérez de Tudela (2009). Investigación cualitativa. Madrid: Esic Editorial.

Brayer Hess, M., \& Linderman, P. (2007). The Expert Expat. Your Guide to Successful Relocation Abroad Moving, Living, Thriving. Londres: Nicholas Brealey Publishing.

Brookfield Global Relocation Service, NFTC y CGHB. (2015). Globally Vocal: Candid insights from Employees Abroad. Recuperado de http://www.cignaglobalhealt. com/.../global-mobility-execsummary-3pdf

Brookfield Global Relocation Service. (2016). Breakthrough to the 
Future of Global Talent Mobility. Global Mobility Trends Survey 2016. Recuperado de http:// globalmobilitytrends.brookfieldgrs.com

Cagigas, J., \& Arribas, J. (2012). ¿Trabajar fuera? Madrid: LID Editorial Empresarial.

Comelles, J., Allué, X., Bernal, M., Fernández-Rufete, J., \& Mascarella, L. (2009). Migraciones y salud. Tarragona: URV Publicaciones.

Deloitte University Press. (2014). Tendencias globales del capital humano 2014. Comprometiendo a la fuerza laboral del siglo XXI. Recuperado de http://www2.deloitte.com/content/dam/Deloitte/ global/Documents/HumanCapital/dttl-hc-trends-spa-spanish.pdf

Espinosa Romero, M. (2009). Los procesos de internacionalización empresarial y la gestión de personas. Madrid: Fundación EOI.

Fernández, E., \& Junkera, B. (2013). Iniciación a los recursos humanos. España, Oviedo: Septem Ediciones S, L.

Gadow, F. (2010). Dilemas: la gestión del talento en tiempos de cambio. Buenos Aires: Granica.

Hart, R. (2012). Preparing for you Move Abroad. Relocating, Settling in, and Managing Culture Shock. Londres: Bravo Ltd.

Hernández, S., Fernández, C., \& Baptista, P. (2010). Metodología de la investigación. México: Mc Graw Hill.

Jiménez, A. (2013). Creando valor... a través de las personas. Una guía para la gestión de personas en el umbral de la e-sociedad. Madrid: Ediciones Díaz de Santos, S.A.

Hodgetts, R., \& Luthans, F. (2003). International Management: Culture, Strategy und Behavior. New York: Mc-Graw Hill Education.

IESE y Ernst \& Young (2008). El proceso de expatriación en empresas multinacionales: visión del expatriado. Recuperado de http://www.iese.edu/en/files/ Informe $\% 20$ Expatriados(para $\% 20$ web) tcm4-38593.pdf

IESE y Ernst \& Young (2011). Políticas de expatriación en multinacionales. Visión de las personas. Recuperado de http://www. iese.edu/research/pdfs/ESTUDIO-156.pdf

IESE-IRCO \& ERES Relocation. (2009). Los retos de la expatriación: aproximación empírica (Estudio \# 86). Recuperado de http: www.iese.edu/research/ pdfs/ESTUDIO-86.pdf

López, A. (2014). Guía práctica para la gestión del trabajador expatriado. España: Bubok Publishing S.L.

Maison, P. (2013). El trabajo en la postmodernidad: reflexiones $y$ propuestas sobre las relaciones 
humanas en los tiempos de la generación $Y$. Buenos Aires: Granica S.A.

ManpowerGroup. (2014). Gestión de talento, un factor clave para acelerar el desempeño empresarial. Tendencias globales, desafios y prioridades. Recuperado de http://www.manpower.cl/ manpower/site/artic/20160208/ asocfile/20160208165953/gestion_de talento.pdf

ManpowerGroup (2015). Encuesta de escasez de talento 2015. Recuperado de https://www.manpowergroup.com.mx/uploads/ estudios/Escasez_Talento_2015. pdf

Marinelli, P., \& Fajardo, K. (2015). Conectados. Estados Unidos: University of South Carolina.

Orsi, A., Bertoia, N., \& BarbosaLima, M (2015). Gestão inter- nacional de recursos humanos. Brasil: Elsevier Editora Ltda.

Porret, M. (2014). Gestión de personas. Manual para la gestión del capital humano en las organizaciones. Madrid: ESIC Editorial.

Rodríguez, A., Fernández, I., \& Romero, J. (2013). La gestión integral de proyectos. Madrid: Pontificia Universidad de Comillas.

Storti, C. (2003). The Art of Coming Home. Yarmouth, ME: Intercultural Press.

Storti, C (2007). The Art of Crossing Cultures. Yarmouth, ME: Intercultural Press.

Wortmann, M (2013). Umgang US-amerikanischer ExpatriateFamilien mit der deutschen Gastkultur. Deutschland: Springer Gabler. 